\begin{tabular}{|c|c|c|}
\hline & International Journal of Current Research in & \\
\hline & Biosciences and Plant Biology & $25^{\circ}$ \\
\hline $\begin{array}{l}\text { EXCELLENT } \\
\text { PUBLISHERS }\end{array}$ & 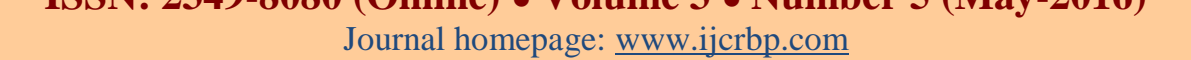 & \\
\hline
\end{tabular}

Case Report

doi: http://dx.doi.org/10.20546/ijcrbp.2016.305.013

\title{
The Hemophilic Dental Patient: A Case Report
}

\author{
Bharati Kolliyavar $^{*}$, Anirudh Acharya ${ }^{2}$ and Srinath L. Thakur3 \\ ${ }^{1}$ Assistant Professor, Department of Periodontics and Oral Implantology, S.D.M. College of Dental Sciences and Hospital, Sattur, \\ Dharwad-580 oo9, Karnataka, India \\ 2Professor, Department of Periodontics and Oral Implantology, S.D.M. College of Dental Sciences and Hospital, Sattur, \\ Dharwad-58o oog, Karnataka, India \\ 3Professor and Principal, Department of Periodontics and Oral Implantology, S.D.M. College of Dental Sciences and Hospital, \\ Sattur, Dharwad-580 0o9, Karnataka, India \\ *Corresponding author.
}

\begin{abstract}
Dental health professionals must be aware of the impact of bleeding disorders on the management of dental patients. The prevalence of hemophilia is estimated to be about $1: 10,000$ birth and that of the severe form of the disease to be about $6 \%$ per $1,00,000$ population. As this is the genetic disorder no complete cure is possible as of now, the only available treatment option is the infusion of factors and some adjuvant therapies depending upon the bleeding conditions. This article presents an overview of hemophilia and smooth management of a hemophilic patient in dental clinic.
\end{abstract}

\section{Article Info}

Accepted: 21 April 2016

Available Online: 06 May 2016

\section{Keywords}

Coagulation factors

Coagulation pathway

Hemophilia

\section{Introduction}

Hemophilia is the disease where there is deficiency of coagulation factors. These are of two types, Hemophilia A and B. Hemophilia A and B are X chromosome linked bleeding disorders included among the rare diseases and caused by mutations in the factor VIII and factor IX genes (Munnucci and Tuddenham, 2001). Both factors take part in the intrinsic pathway of blood coagulation and affected individuals have severe, moderate and mild forms of the diseases, defined by factor levels of $1 \%$ or less, 2 to $5 \%$ and 6 to $40 \%$ respectively. The prevalence of Hemophilia A is 1 in 5000 male live births, and that of hemophilia B is 1 in 30,000 (Munnucci and Tuddenham, 2001; BoltonMaggs and Passi, 2003). Individuals with a factor level < $1 \mathrm{IU} / \mathrm{dL}$ are classified as severe hemophiliacs and represent about half of the diagnosed cases. Subjects with factor levels between 1-5 IU/dL have moderate and mild hemophilia (Coppola et al., 2010). Hemophilia A and B are difficult to distinguish from a clinical point of view. Replacement of haemostatic concentrations of the deficient factor is the mainstay of treatment of bleeding episodes. Recurrent joint bleeds inevitably leading to crippling arthropathy (Rosendaal and Lafeber, 2006), were the hallmarks of the disease before the 1970s, when plasma fractions containing FVIII or FIX were still not available. At that time mortality from bleeding was high, and the life expectancy of persons with hemophilia was much lower than that of general population (Munnucci, 2008; Plug et al., 2004).

The discovery of cryoprecipitate in 1964 and the subsequent development of lyphophilized factor concentrate in the 1970s paved the way for modern effective hemophilia replacement therapy and enabling severe hemophilia to treat joint bleeds as early as possible. This was associated with a significant reduction in morbidity. However the most important advance was recombinant gene technology and Protein purification techniques, which enable the development of highly purified recombinant FVIII and FIX products (Franchini et al., 2009). 
Very few reports are available in the literature regarding hemophilic patients's management in the dental office. Preoperative laboratory test of the hemostatic system are-

- Bleeding time to determine platelet function (normal range: 2-7 min).

- Activated partial Thromboplastin time to evaluate the intrinsic coagulation factor (normal range is $25 \pm 10 \mathrm{sec}$ ).

- International normalized ratio to measure the extrinsic pathway (normal range is 1).

- Platelet count to quantify platelet function (normal range is $150,0000-450,0000 \mu \mathrm{l}$ ).

\section{Case report}

A 38 year old male patient reported to the Department of Periodontology, SDM College of Dental Sciences and Hospital, Dharwad, (Karnataka) with the chief complaint of loose teeth in the lower front region of the jaw since 1 year, and he also complained of bleeding in the same region since 6 days. Patient is a hemophilic since childhood and is on supplementation of factor VIII. Patient gave a history of prolonged bleeding from minor cuts, and bleeding from the knee joints. Patient had taken FVIII of 2780 Units on the previous morning.

Patient's family history revealed mother to be a hemophilic carrier, his Aunt (mother's sister) is also a hemophilic carrier. Patient's cousin brother is a hemophilic sufferer. Examination of oral cavity revealed calculus and subgingival deposits, recession with lower anteriors, generalized pockets, decayed 18, 28, 46, 38 root stumps with 18 grade III mobile 41, grade II mobile 42 , grade I mobile 31 . Investigations such as bleeding time, clotting time, and partial thromboplastin time were carried out.

Treatment included supragingival scaling which was done, keeping injection Tranostat one Ampule and the local measures to prevent bleeding (Gel foam, gauze, etc.) in vicinity. Patient was referred to the oral surgery department were 41 and 38 were extracted, figure of 8 suture was placed to approximate the gingiva in the 38 region and for Hemostasis. Post surgical instructions were given. Patient was observed for half an hour post extraction, no bleeding was seen. Patient was prescribed tablet Tinimox (Amoxycillin semisynthetic broad spectrum beta lactum antibiotic) three times a day for 5 days and acetaminophen tablet for 4 days. Patient responded well to the non surgical periodontal therapy, and the gingival inflammation was reduced.

\section{Discussion}

The disease is known since ancient times but Schonelein in 1839 gave this bleeder's disease its present name as Hemophilia. The frequency of hemophilia varies in different races, the highest incidence being in populations of Britan, Northern Europe and Australia. Western literature reports give an overall incidence of Hemophilia in one in 10,000 male births. Another interesting facet of the hemophilia which has attracted investigators and researchers is the occurrence of this disorder in the royal blood of Great Britain and some European Royal families.

Another interesting facet of the hemophilia which has attracted investigators and researchers is the occurrence of this disorder in the royal blood of Great Britain and some European Royal families.

Factor VIII is synthesized in Hepatic parenchyma cells and regulates the activation of factor $\mathrm{X}$ in intrinsic coagulation pathway. Factor VIII circulates in blood complexed to another larger Protein, von Will brand's factor (v WF), which comprises $99 \%$ of the factor VIIIVwf).

The transmission of Hemophilia to the next generation can be prevented by the following methods.

1. Prenatal intrauterine diagnosis with termination of pregnancy as an option

2. Pre implantation genetic diagnostic testing (PGD)

3. IVF with egg or sperm donation (Sona and Lingam, 2010).

To prevent surgical hemorrhage factor VIII levels of at least $30 \%$ are needed. Three methods of replacement therapy have been employed to maintain circulating factor levels above the $20 \%$ minimum necessary for hemostasis during surgical and healing phases. These include intermittent replacement therapy, continuous intervenous factor infusion therapy, and single preoperative factor concentrate infusion combined with an antifibronytic mouth wash (Patton, 2003).

Patients with coagulopathies may neglect their oral health due to fear of bleeding during tooth brushing and flossing, which leads to increased gingivitis, periodontitis and caries. Periodontal probing, supragingival scaling and polishing can be done normally without the risk of significant bleeding. Factor replacement is seldom 
needed for subgingival scaling and root planning if these procedures are done carefully. Ultrasonic instrumentation may result in less tissue trauma. For severely inflamed tissues, initial treatment with Chlorhexidine mouthwashes and gross debridement is recommended to reduce tissue inflammation before deep scaling (Webster and Courtney, 1968).

Factor replacement may be required before extensive periodontal surgery and use of nerve blocks. Periodontal surgical procedures warrant elevating circulating factor levels to $50 \%$ and use of post-treatment antifibrinolytics. Periodontal packing materials and custom vinyl mouth guards (stents) are used to aid in hemostasis and protect the surgical site, but these can be dislodged by severe hemorrhage or subperiosteal hematoma formation (Patton, 2003).

Antifibrinolytic agents may be incorporated into periodontal dressings for enhanced effect. Post treatment antifibrinolytic mouthwashes are usually effective in controlling protracted bleeding (Gupta et al., 2007). Patients with bleeding disorders, appropriately prepared preoperatively, are generally as able to withstand dental care as well as unaffected individuals. Consultation with the patient's physician is recommended for guidance on medical management of higher risk surgical dental management (Patton, 2003). Tranexamic acid (Cyclokapron) is currently the antifibrinolytic of choice. The dose is $15-20 \mathrm{mg} / \mathrm{kg}$ orally, three times a day. For dental extraction complicated by infection or abscess formation, or when the patient is HIV positive, the use of prophylactic antibiotic therapy is recommended. The patient may remain in the hospital at least overnight after dental extraction so that therapy can be supervised and the patient carefully monitored, however this may not be possible always. Major or multiple extractions may require a hospital stay of two or three nights, although it is more likely that the patient would return to the hemophilia centre as an outpatient (AHCDO, 2005).

Many patients do not even receive non-surgical comprehensive periodontal therapy because practioners do not know how to address coagulation disorders (Nickles et al., 2010). Very few reports are available in the literature regarding hemophilic patient's management in the dental office. The patient reported in this case was effectively managed in the dental clinics without any untoward complications. Since it is difficult to distinguish between Hemophillia A and B, patient's awareness of the disease plays an important role as a supplement in dental management as out-patient. This case also shows that managing hemophilia in the dental office can be effective if a detailed history of the patient is recorded. Furthermore, the required armamentarium, including the drugs of choice should be available in the dental office.

\section{Conclusion}

In the past three decades, hemophilia has moved from the status of neglected and often fatal heriditory hemorrhagic disorder to that of a defined group of well characterized molecular entities. Among the most prevalent monogenic disorders (cystic fibrosis, thalassemia, muscular dystrophy) hemophilia enjoys the most efficacious and safe treatment. Indeed after the dramatic events of widespread blood borne virus transmission in the 1970s-1980s, there has been a strong drive towards a continuous improvement in the efficacy and safety of replacement therapy and towards the cure of the disease through gene therapy. Dental practitioners need to remember that successful management of such patients is possible even in emergency situations if all requirements are met within the dental operatory and non surgical periodontal therapies can be effectively managed in the dental clinics.

\section{Conflict of interest statement}

Authors declare that they have no conflict of interest.

\section{References}

AHCDO, 2005. Guidelines for the Management of Patients with Haemophilia undergoing Surgical Procedures. Australian Haemophilia Centre Directors Organisation July 2005.

Bolton-Maggs, P.H., Passi, K.J., 2003. Hemopholia A and B. Lancet. 361, 1801-1809.

Coppola, A., Capua, M. D., Dario, M. N., Minno, D., Paulo, M. D., Marrone, E., et al. 2010. Treatment of hemophilia: A review of current advances and ongoing issues. J. Blood Med. 1, 183-195.

Franchini, M., Coppla, A., Molinari, A.C., 2009. Forum on the role of recombinant factor VIII in children with severe hemophilia A. Hemophilia. 15, 578-585.

Gupta, A., Epstein, J. B., Cabay, R. J., 2007. Bleeding disorders of importance in Dental care and related patient management. JCDA. 73, 77-83.

Munnucci, P.M., Tuddenham, E.G.D., 2001. The hemophiliac from royal genes to gene therapy. N. Engl. J. Med. 344, 1773-1779.

Munnucci, P.M., 2008. Back to the future: A recent history of hemophilia treatment. Hemophilia. 14, 10-18. 
Nickles, K., Wohlfeil, M., Alesci, S., Miesbach, W., Eickholz, P., 2010. Comprehensive treatment of periodontitis in patients with von Willebrand disease. J. Periodontol. 81, $1433-1440$.

Patton, L.L., 2003. Bleeding and clotting disorders. In: Burket's Oral Medicine: Diagnosis and Treatment. $10^{\text {th }}$ Edn. BC Decker, Hamilton (ON). pp.454-477.

Plug, I., Van der Bom, J.G., Peters, M., 2004. Thirty years of hemophilia treatment in Netherlands, 1972-2001. Blood. 104, 3494-3500.
Rosendaal, G., Lafeber, F.P., 2006. Pathogenesis of hemophilic arthropathy. Hemophilia. 12, 117-121.

Sona, P.S., Lingam, C. M., 2010. Hemophilia an overview. Int. J. Pharmaceut. Sci. Rev. Res. 5, 18-26.

Webster, W.P., Courtney, R.M., 1968. Diagnosis and treatment of periodontal disease in the hemophiliac. In: Proceedings of Dental Hemophilia Institute. National Hemophilia Foundation, New York. 288p.

\section{How to cite this article:}

Kolliyavar, B., Acharya, A., Thakur, S. L., 2016. The hemophilic dental patient: A Case report. Int. J. Curr. Res. Biosci. Plant Biol. 3(5), 84-87. doi: http://dx.doi.org/10.20546/ijcrbp.2016.305.013 